\title{
"Alle Hilfsmittel an der Hand": note sulle prime fotografie collezionate da Aby Warburg
}

\begin{abstract}
There is no evidence of a consistent theoretical position of Aby Warburg regarding photography, but his scattered notes on the subject allow for a deductive evaluation. The considerable use of photographs that he made in his work suggests a wide range of methodological approaches, with significant implications for the disciplinary and methodological definition of art history and the development of photographic documentation at the turn of the $20^{\text {th }}$ century. This essay provides examples of Warburg's early attention to photography, both as a research tool and a required piece of equipment for any research institute.
\end{abstract}

\section{Keywords}

WARBURG, ABY M.; BIBLIOTHEK WARBURG; DOCUMENTARY

PHOTOGRAPHY; NAYA, CARLO; ALINARI; PIDERIT, THEODOR;

DARWIN, CHARLES

$\mathbf{N}$

on abbiamo notizia di una posizione teorica di Aby Warburg rispetto alla fotografia, ma solo di note sparse, di diversa natura, da cui trarre valutazioni per deduzione. L'ampio ricorso alle fotografie nel suo lavoro, tuttavia, offre una gamma di casi e di approcci che vanno considerati nel quadro del dibattito, avviato tra fine Ottocento e inizio Novecento, sulla definizione disciplinare e metodologica della storia dell'arte e sullo sviluppo del settore di documentazione fotografica di monumenti architettonici e opere d'arte pittoriche e scultoree ${ }^{-1}$.

Le fotografie più famose di Warburg, raccolte e realizzate nel 1895-1896 durante il viaggio presso i villaggi Pueblo in New Mexico e Arizona, sono state discusse nel contesto degli studi culturali e nella prospettiva dell'antropologia in relazione alla storia dell'arte ${ }^{-2}$. La nota che segue mira invece a offrire esempi dell'interesse di Warburg per le riprese fotografiche di opere d'arte, mostrando la precoce attenzione dello studioso alla fotografia come indispensabile strumento per la ricerca 
storico-artistica e come dotazione necessaria di un istituto dedicato allo studio delle immagini ${ }^{-3}$. Gli esempi permettono altresì di evidenziare - o isolare -, alle origini dell'impresa scientifica warburghiana, questioni cruciali che riguardano il valore documentale delle immagini nella ricerca interdisciplinare, lo statuto della fotografia di opere d'arte, il potenziale di disseminazione e veicolazione intermediale delle fotografie. Nell'inverno 1888-1889 il ventiduenne Aby M. Warburg si trovava a Firenze al seguito di August Schmarsow, il quale, previo congedo per il semestre invernale dagli obblighi di professore straordinario di storia dell'arte a Breslavia, aveva qui trasferito le sue lezioni (fig. 1). Le sue conferenze pubbliche si tenevano al Circolo filologico di Palazzo Ferroni, mentre altre lezioni sulla scultura italiana e la relazione tra Masolino e Masaccio avevano luogo nel suo appartamento privato. Si dava così inizio alle attività di quello che sarebbe divenuto, di lì a pochi anni, il Kunsthistorisches Institut in Florenz ${ }^{-4}$. Nel 1893, nel corso del secondo congresso di storia dell'arte a Norimberga, veniva creato il comitato esecutivo e nella "Kunstchronik" veniva diffuso un Appello per la fondazione di un Istituto di Storia dell'arte a Firenze, il cui primo intento era la

Anschaffung einer möglichst vollständigen kunstwissenschaftlichen Bibliothek und einer großen Sammlung von zu vergleichenden Studien geeigneten Abbildungen, welche vereinigt in passenden Arbeitsräumen aufgestellt und bequemer Benutzung zugänglich gemacht werden ${ }^{-5}$.

$-$

All'indomani della costituzione formale dell'istituto nel 1898 nasce il "Verein zur Förderung des Kunsthistorischen Instituts in Florenz", con lo scopo di sostenere con finanziamenti statali e privati le sue attività: nel 1899 l'istituto vanta già una collezione di 1.900 volumi e 5.000 immagini, tra fotografie e altri materiali visivi. Per quanto riguarda invece la biblioteca di Aby Warburg, essa diventa formalmente istituto di ricerca nel 1921, ma la collezione ha origine già nei primi anni del percorso di formazione dello studioso ${ }^{-6}$. Affatto diversa come impresa - non da ultimo dal punto di vista del finanziamento, partecipato nel caso dell'istituto fiorentino, privato in quello warburghiano - anche l'amburghiana Kulturwissenschaftliche Bibliothek Warburg (KBW) - ${ }^{7}$, così nominata dal 1926, vede l'associazione necessaria fra strumenti diversi, quali libri e immagini, così come riportato in documenti relativi alle origini delle sue raccolte. I fogli di "Jahrestabellen", diagrammi colorati su carta millimetrata che indicano l'andamento delle acquisizioni a partire dal 1886, anno di iscrizione dello studioso all'Università di Bonn, documentano, inoltre, già a partire dal 1888, l'importanza attribuita alla sezione visiva della collezione anche in termini di spesa: tanto che se tra i costi sono indicati Bücher (libri), Bucheinband (legatoria), Spesen (spese varie), una voce distinta è per Abbildungen (illustrazioni) ${ }^{-8}$. Anche i diari personali di Warburg e le lettere alla famiglia confermano la pratica della raccolta di libri e fotografie e, allo stesso tempo, 

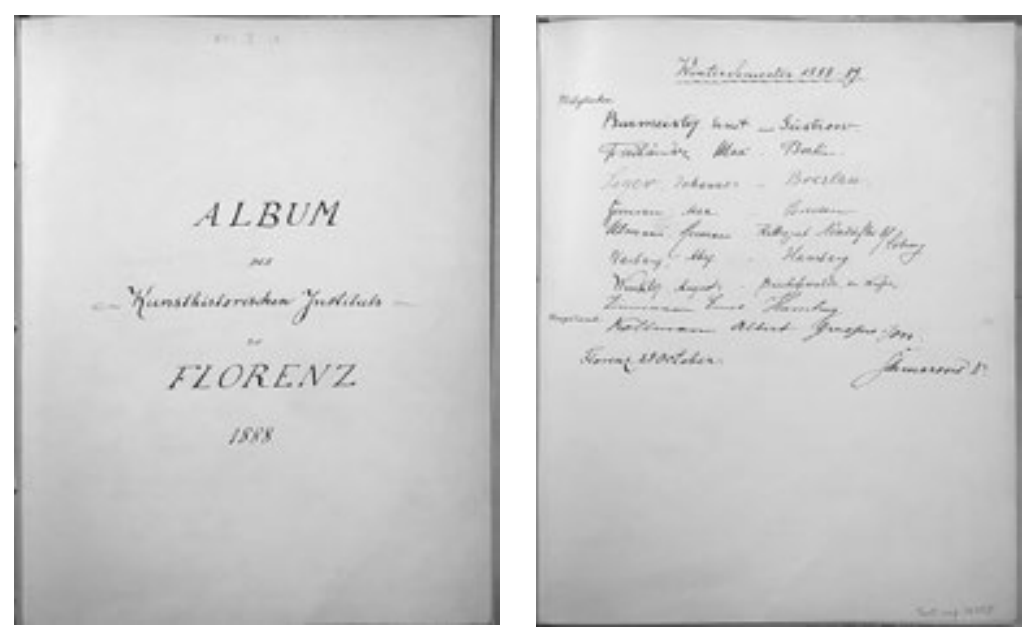

Album des

Kunsthistorischen

Instituts zu Florenz,

Wintersemester

1888-1889,

28 October 1888, pp. 1

(frontespizio) e 3

(elenco dei partecipanti al

seminario di Schmarsow).

Archivio del

Kunsthistorisches Institut

in Florenz-Max Plank

Institut

attestano la consapevolezza dell'aspetto letteralmente imprenditoriale della fondazione di una biblioteca specializzata. Leggiamo in due lettere del gennaio 1889:

Ich muss hier den Grundstock zu meiner Bibliothek und Photographiensmlg[Sammlung] legen und beides kostet viel Geld und repräsentiert bleibenden Werth ${ }^{-9}$.

\section{E ancora:}

[...] ich muss eben alle Hilfsmittel (Bücher, Phot.) an der Hand haben $-{ }^{10}$.

Nel 1933 la Kulturwissenschaftliche Bibliothek Warburg sarà trasferita a Londra non solo per salvare dal nazismo l'eredità intellettuale di Aby Warburg, morto nell'ottobre 1929, ma anche la sorte di diversi studiosi. Dopo numerose traversie e spostamenti, nel 1944 la biblioteca entrerà a far parte degli istituti della University of London con la denominazione di Warburg Institute, infine nel 1958 verrà ricollocata nell'attuale edificio in Woburn Square, a Bloomsbury ${ }^{-11}$. Biblioteca e collezione fotografica del Warburg Institute di Londra preservano ampie tracce ed evidenze del momento della fondazione, ossia libri e fotografie del primo nucleo della collezione ${ }^{-12}$. Tra queste ultime, chi scrive ha identificato tre stampe all'albumina, due da opere fiorentine - Lippi in Santa Maria Novella e Donatello in Orsanmichele - e una dagli affreschi di Giotto alla Cappella Scrovegni di Padova. Le tre fotografie recano sul verso un palinsesto di 'metadati' di contenuto e strutturali, tra i quali si intravede un elemento importante che può aiutare a collocare il loro arrivo nella collezione di Warburg e, allo stesso tempo, a testimoniare l'interesse dello studioso per questo genere di opere artistiche e per la loro traduzione a stampa. Si tratta di timbri posti sul verso originale 
delle tre fotografie, e che oggi si possono cogliere solo collocandole in controluce poiché la telatura con cui sono state condizionate ne impedisce la leggibilità. Scorgiamo così il timbro personale usato da Warburg in questi anni per segnare le proprie acquisizioni e le annotazioni manoscritte che registrano luogo e data di acquisizione (figg. 2-4) ${ }^{-13}$.

Alcune note sono necessarie per contestualizzare queste stampe all'albumina negli studi sulla fotografia di documentazione dell'arte. In questa prospettiva, il giudizio critico sulla qualità documentaria di ripresa e traduzione da opere d'arte è legato, prima ancora che alle retoriche di oggettività - o neutralità interpretativa - della fotografia, a una sintassi tecnico-operativa. Negli anni Ottanta dell'Ottocento, quando Warburg acquistò queste fotografie, la ripresa di opere d'arte era ancora limitata da diverse questioni che comprendevano i limiti dati dalle emulsioni e lo stato di conservazione delle opere, ma soprattutto la possibilità di gestire l'ingombro della strumentazione fotografica, la scelta di punti di ripresa ottimali, lo spostamento delle opere stesse (come per i dipinti) anche per risolvere i problemi legati all'illuminazione. Nel caso delle fotografie degli affreschi, abbiamo due esempi di traduzione fotografica in monocromia e relative incongruenze nella traduzione dei valori chiaroscurali del colore della pittura, dettati dalle sostanze fotosensibili disponibili allora per preparare le lastre negative. I materiali fotografici erano ancora particolarmente sensibili alla luce blu, che si traduceva in toni molto chiari nella stampa in positivo, mentre al contrario i colori rosso e verde erano restituiti con grigi molto più scuri. Gelatina e lastre ortocromatiche, sensibili anche al verde, furono commercializzate nel 1882; nel 1903 furono prodotte le prime emulsioni sensibili alla luce arancione; dal 1905 alla luce rossa; le prime lastre effettivamente pancromatiche arrivarono solo nel $1906{ }^{-14}$. Questi dati sono cruciali nella storia della fotografia dei dipinti e per il pensiero sulla vita intermediale - analogica - delle immagini di opere di pittura.

La scultura (non dipinta) non pagava questo limite nella traduzione del colore. Per la fotografia delle arti plastiche, e particolarmente il tutto tondo, si assiste difatti al precoce sviluppo di un dibattito sull'estetica della ripresa, nei termini della correttezza del punto di vista, del taglio e delle ombre, della visione e interpretazione di volumi e raffigurazioni. È il caso del lavoro seminale di Heinrich Wölfflin Wie man Skulpturen aufnehmen soll del $1897^{-15}$. Le qualità dello 'stiacciato prospettico' donatelliano (fig. 2), ad esempio, sono qui isolate nel taglio dell'inquadratura fotografica, tradotte e proiettate nella bidimensionalità della stampa, evidenziate dalle caratteristiche chiaroscurali del monocromo di un'albumina di fine Ottocento. In questi termini, la fotografia appare un ausilio interpretativo - già condizionato da tecnica ed estetica della ripresa - oltre che un supporto alla memoria ${ }^{-16}$ e uno strumento per la comparazione.

Nel caso della fotografia della Strage degli innocenti agli Scrovegni possono essere fatte considerazioni di diversa natura. L'immagine (fig. 3), firmata e numerata sulla lastra negativa "C. Naya 20", fa parte 


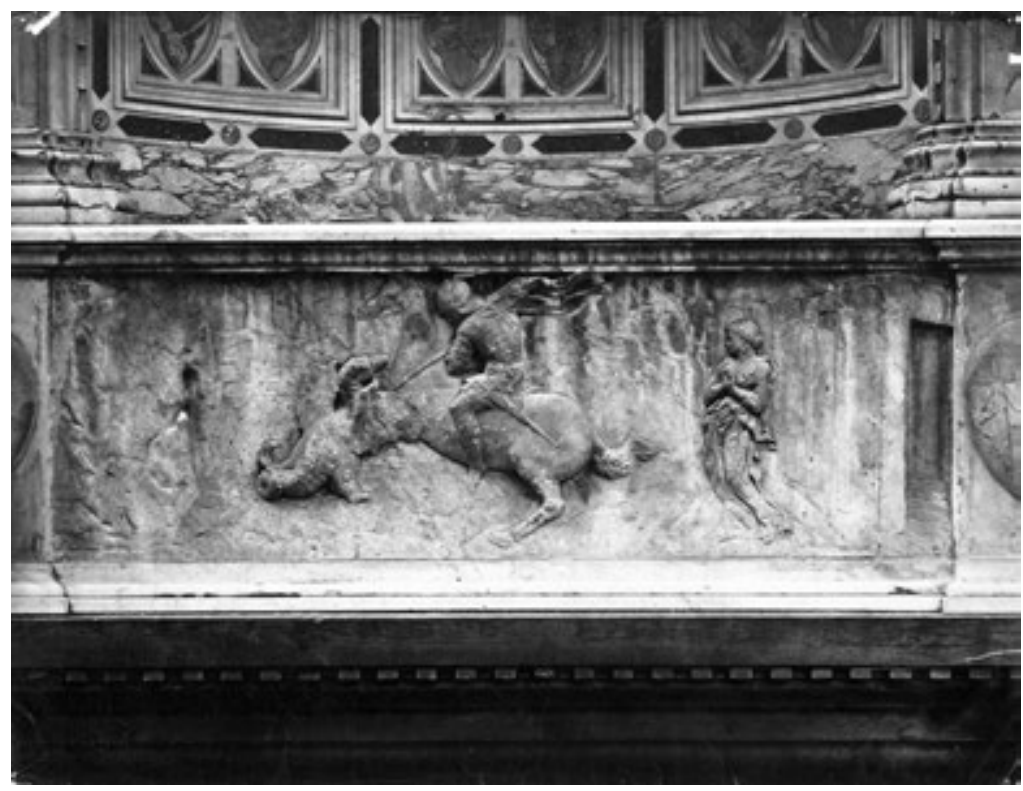

a.

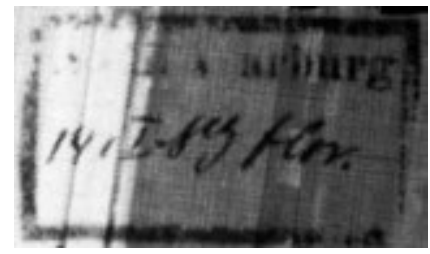

b.

della prima serie di riprese mai realizzata degli affreschi di Giotto. Si tratta, altresì, della prima serie di fotografie da opere d'arte che Carlo Naya trasse dal vero anziché da stampe o disegni - pratica, quest'ultima, comune e diffusa agli albori della fotografia documentale d'arte, per le difficoltà tecniche più sopra tratteggiate e in continuità con una consolidata tradizione e consuetudine visiva alle tecniche di traduzione grafica ${ }^{-17}$. La serie venne realizzata tra il 1863 e il 1865 ; le prime 13 fotografie vennero incluse nei cataloghi del 1865 e del 1870 quando la serie completa venne rinumerata da 1 a 59 , includendo le Storie di Anna, Gioachino e Gesù, e Virtù e Vizi ${ }^{-18}$. Per oltre trent'anni queste furono le uniche riprese degli affreschi degli Scrovegni disponibili sul mercato. La serie è stata di recente correttamente identificata, datata e interpretata nel contesto dei restauri degli affreschi, della loro fortuna critica di fine Ottocento e dell'avvedutezza di Naya rispetto alla richiesta di un mercato di amatori e di studiosi ${ }^{-19}$. È infatti mutuo e reciproco lo scambio tra produttore e fruitore della fotografia. Gli storici dell'arte compravano fotografie ordinandole dai cataloghi e perciò attingendo da repertori già determinati. Opere, soggetti, dettagli erano altresì ripresi su commissione di storici, critici, conservatori d'arte a ditte specializzate

\section{Fotografo non} identificato,

a. “Donatello, San Giorgio e il drago", 1889 (ante quem).

Stampa all'albumina, supporto primario $19 \times 25$ $\mathrm{cm}$ (supporto secondario di tela $19 \times 25 \mathrm{~cm}$ ). London, Photographic Collection of the Warburg Institute.

b. Timbro e nota manoscritta sul verso della fotografia telata, in basso a destra: "A.[by] M.[oritz] Warburg / 14.I.89 / Flor.[enz]" 


\section{Carlo Naya,}

a. "Giotto, Strage degli innocenti", 1863-65.

Stampa all'albumina, supporto primario $18 \times 24$ $\mathrm{cm}$ (supporto secondario di tela $18 \times 24 \mathrm{~cm}$ ). London, Photographic Collection of the Warburg Institute.

b. Verso della fotografia telata, con timbro e nota manoscritta in alto a destra: "A.[by] M. [oritz] Warburg / 28.1.89 / Flor.[enz]"

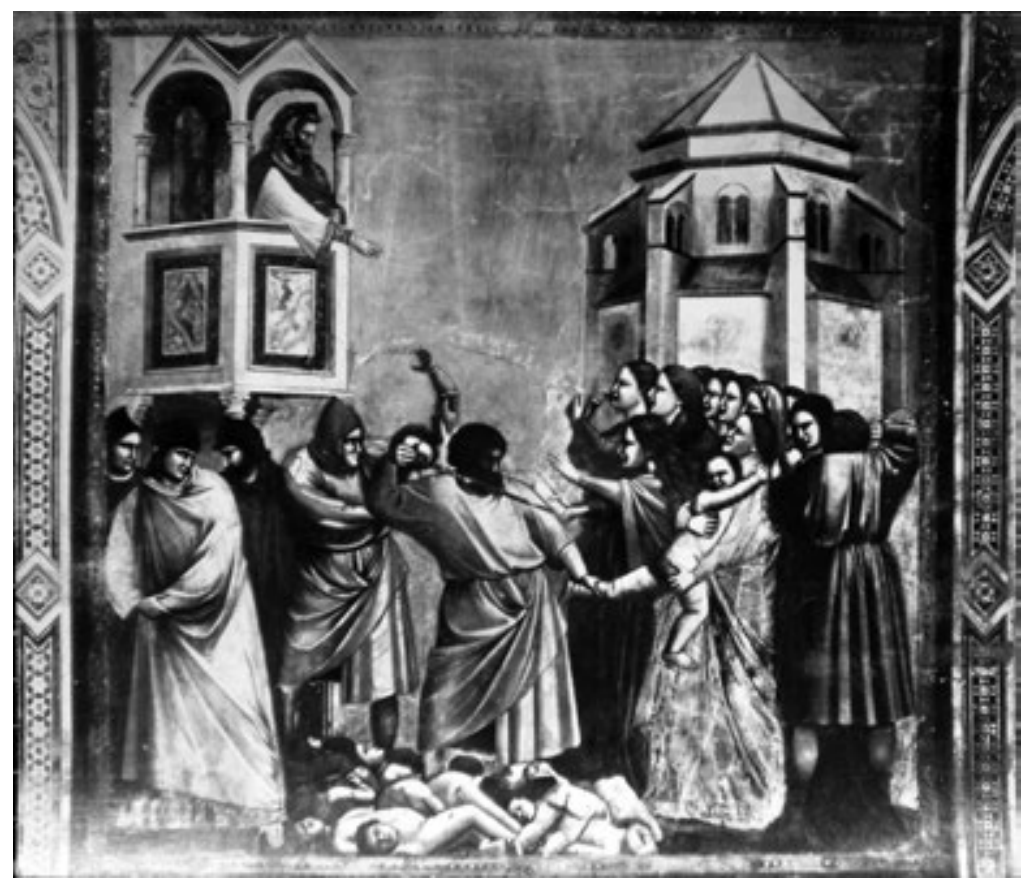

a.

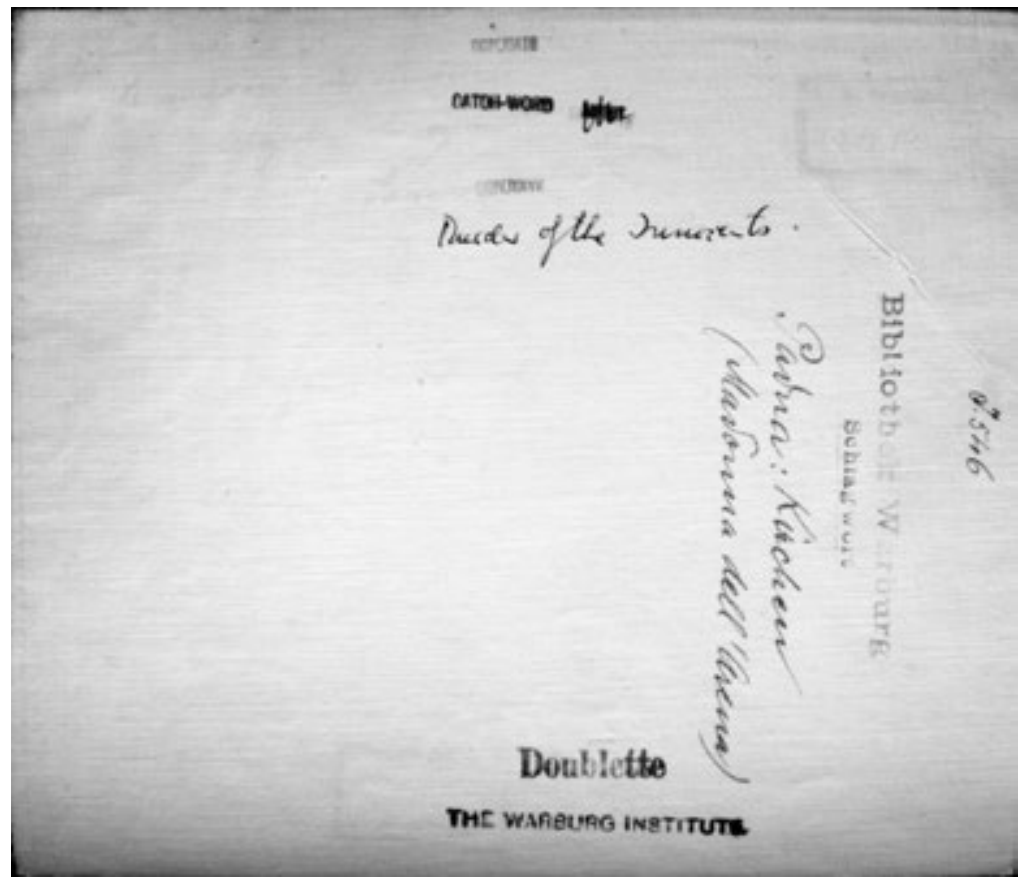

b. 


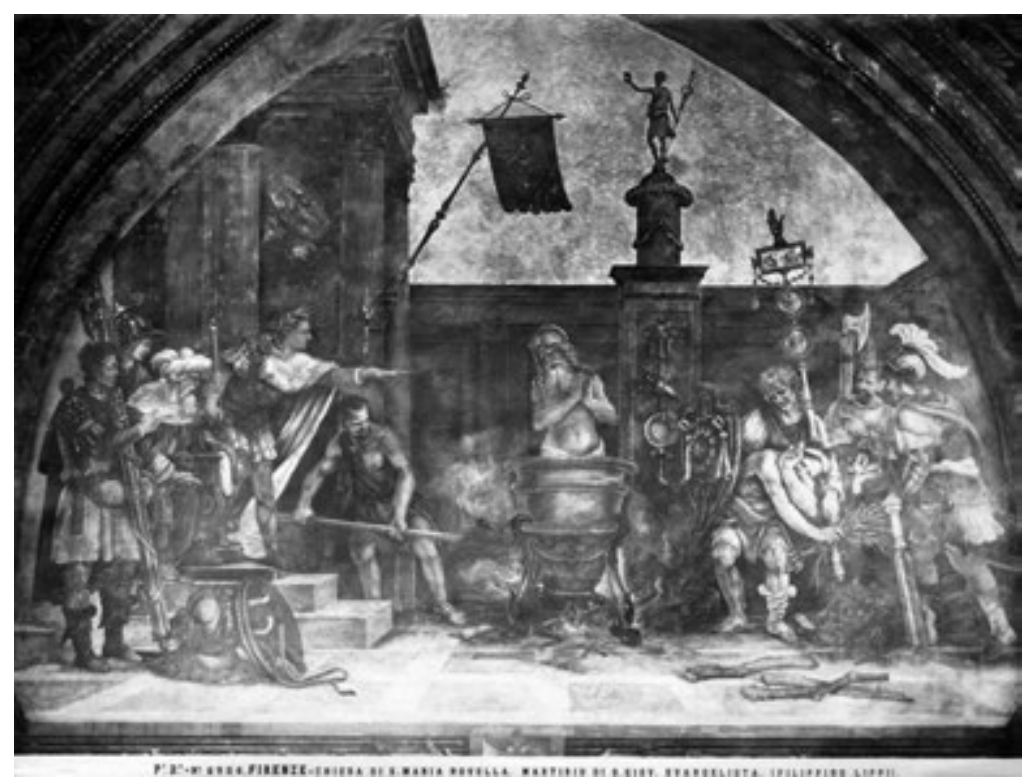

a.

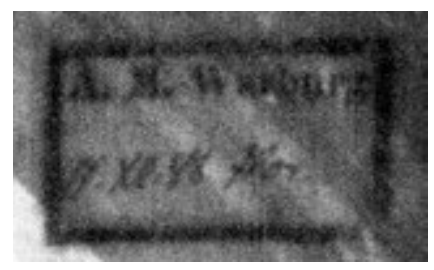

b.

o a fotografi indipendenti. Le fotografie, eseguite seguendo precise prospettive critiche e di ricerca e risolvendo dati problemi tecnici, in alcuni casi erano poi immesse sul mercato, (e) o veicolate a mezzo pubblicazione, (e) o incluse nelle collezioni. Si può considerare, a titolo di esempio, lo studio di Aby Warburg sui ritratti nelle Storie di San Francesco affrescate da Domenico Ghirlandaio nella Cappella Sassetti in Santa Trinita a Firenze ${ }^{-20}$. Warburg pubblica le prime "grössere Detailaufnahme[n] ${ }^{-21}$ di alcuni personaggi della cerchia medicea ritratti con Francesco Sassetti in uno degli episodi delle Storie. Di più: quattro delle fotografie pubblicate come tavole a corredo del suo saggio sono riprese per la prima volta da Alinari su sua "Veranlassung" (richiesta) -22. Le immagini entrano nel catalogo della ditta con le identificazioni proposte da Warburg, tanto che tra fine 1901 e inizio 1902 in alcune lettere per le bozze e le copie recensione del saggio, lo studioso si dice ansioso di vedere il lavoro pubblicato, dato che Alinari avrebbe a breve venduto le fotografie singolarmente ${ }^{-23}$. Da un lato, le prime fotografie di opere d'arte dotate di una qualità documentaria adeguata a supportare lo studio e la ricerca condizionavano al contempo le possibilità e le modalità di accesso, percezione e visione dell'opera ${ }^{-24}$; dall'altro, la nascita di
Alinari

a. P. $2 .^{A}-N .^{\circ} 6908$.

Firenze - Chiesa di S.

Maria Novella. Martirio di S. Giov. Evangelista

(Filippino Lippi), 1873-76.

Stampa all'albumina,

supporto primario $18 \times 25$

$\mathrm{cm}$ (supporto secondario

di tela $18 \times 25 \mathrm{~cm}$ ).

London, Photographic

Collection of the Warburg Institute.

b. Timbro e nota manoscritta sul verso della fotografia telata, in alto a destra: “A.[by] M.[oritz] Warburg / 17.XII.88 / Flor.[enz]" 
un vero dibattito storico-artistico e il profilarsi di una definizione disciplinare contribuivano in modo determinante all'evoluzione dello strumento fotografico, dal punto di vista dell'applicazione tecnica come da quello estetico. Possiamo inoltre dire che Warburg si procurò, con la fotografia di Naya, e commissionò, con le fotografie Alinari dei ritratti in Santa Trinita, il corrispettivo visivo di incunaboli: queste riprese rappresentano il punto di inizio della biografia inter-mediale analogica degli affreschi, o meglio, delle immagini - nel senso di visione - degli affreschi giotteschi e dei dettagli dagli affreschi di Ghirlandaio.

La fotografia del Martirio di San Giovanni Evangelista di Filippino Lippi (fig. 4) ha il numero di inventario 6.908 del catalogo Alinari 1876, appendice al catalogo generale 1873 . La ripresa è pertanto da collocare tra queste due date ${ }^{-25}$. Anche in questo caso è in gioco la qualità interpretativa della ripresa fotografica, condizionata tecnicamente ed esteticamente. Nel lavoro di documentazione dell'arte degli Alinari è stata riconosciuta, ad esempio, un'attenzione condizionata da incidenze narrative e focalizzazioni ritrattistiche dalle Vite di Giorgio Vasari - ${ }^{26}$. Nello specifico, possiamo aggiungere che, ad oggi, questa fotografia acquisita nel dicembre 1888 risulta essere la più precoce nel nucleo warburghiano della Photographic Collection del Warburg Institute. Considerando che la corrispondenza warburghiana registra la prima occorrenza di "Alinari” nel $1890^{-27}$, la stampa documenta anche uno dei primi contatti diretti di Warburg con questo stabilimento, da far coincidere con il seminario fiorentino al seguito di Schmarsow ${ }^{-28}$. La data segnata sulla fotografia e l'identificazione trovano infatti riscontro anche nei diari di Warburg, che il 12 dicembre 1888 menziona proprio una visita ad Alinari e, tra altri artisti e soggetti considerati nelle riproduzioni disponibili, Filippino Lippi ${ }^{29}$.

Questi tre oggetti fotografici costituiscono per il giovane allievo di Schmarsow non solo aide-mémoire delle opere viste dal vivo o anche solo immaginate (come Giotto a Padova), ma anche altrettanti strumenti di studio sui gesti e le espressioni nell'arte. Lo studioso elaborava proprio in quel semestre invernale 1888-1889 un Progetto di critica del Laocoonte a partire dall'arte del Quattrocento a Firenze, discutendo "lo sviluppo del pittorico nei rilievi di Ghiberti", caso studio per un seminario da tenere a Bonn il maggio successivo ${ }^{-30}$. Il riferimento è il Laocoonte di Lessing; il concetto di "pittorico" nelle arti plastiche era discusso in relazione alla scelta del momento nella rappresentazione figurativa e a temi cardine dell'estetica ottocentesca, come l'ekphrasis e il "transitorio". Esempio 'classico', in questo senso, è la contrapposizione tra la sequenza dei versi poetici di Virgilio che narrano la morte del sacerdote troiano e il 'fermo immagine' a tutto tondo del Laocoonte capitolino, colto dai maestri rodî nell'istante del morso del mostro marino che stritola le tre figure del gruppo scultoreo. Nel particolare caso poi dello 'stiacciato' alla base del San Giorgio (fig. 2), il lavoro di Warburg si concentra sull'analisi di elementi pittorici del rilievo scultoreo come, ad esempio, l'uso della prospettiva ed evidenzia il primo piano della 


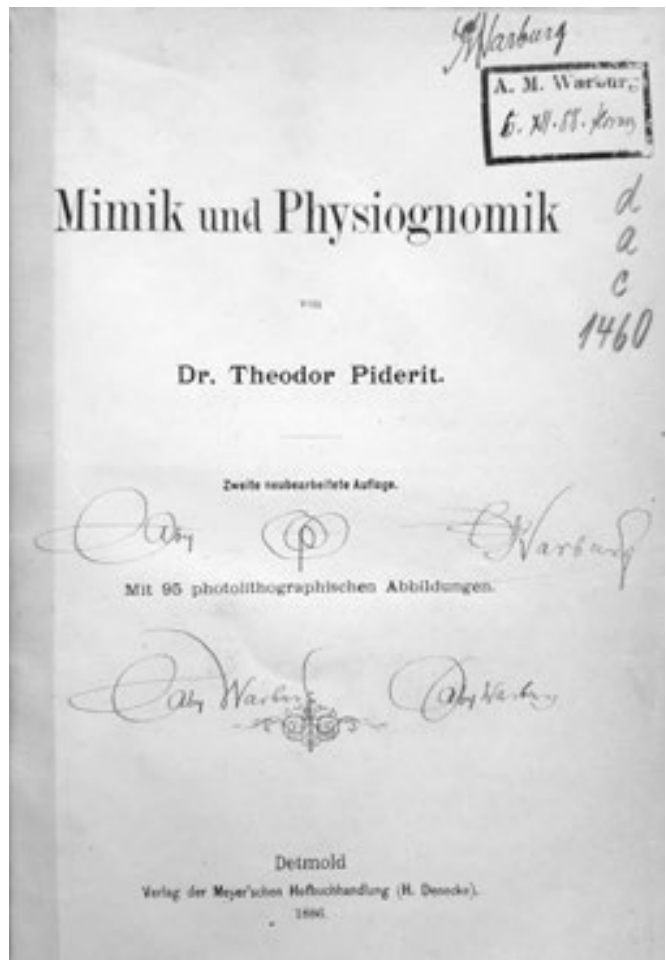

05

Frontespizio del volume

Theodor Piderit, Mimik

und Physiognomik,

Detmold, Verlag der

Meyer'schen

Hofbuchhandlung

(H. Denecke), 1886.

Library of the Warburg

Institute.

Timbro e nota

manoscritta in alto a

destra: “A.[by] M.[oritz]

Warburg / 6.XII.88 /

Florenz]"

06

14. Laocoonte (Senkrechte mit horizontalen

Stirnfalten). Julius

Klinkhardt in Leipzig,

tavola fotolitografica

da Theodor Piderit, Mimik

und Physiognomik,

Detmold, Verlag

der Meyer'schen

Hofbuchhandlung

(H. Denecke), 1886, p. 86 
Carta di guardia

di Charles Darwin,

Der Ausdruck der

Gemüthsbewegungen

bei dem Menschen

und den Thieren,

E. Schweizerbart'sche

Verlagshandlung,

Stuttgart, 1872.

Library of the Warburg

Institute.

Timbro e nota

manoscritta in alto a

destra: “A.[by] M.[oritz]

Warburg / Hbg

[Hamburg] Ostern 89"

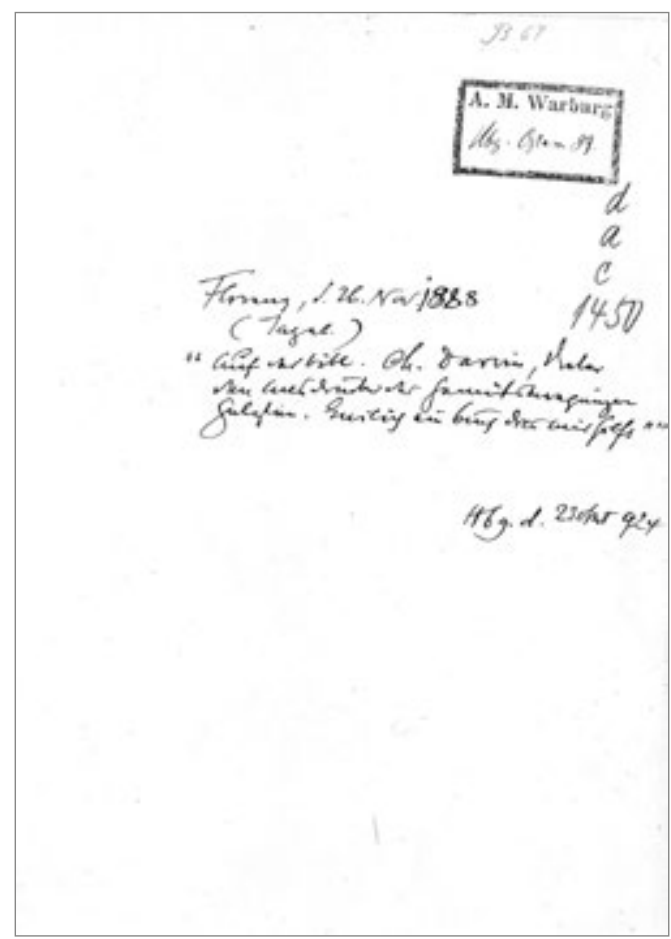

rappresentazione all'altezza dell'occhio dell'osservatore - la nicchia in Orsanmichele - e convergente sullo sfondo della scena - San Giorgio che salva dal drago la principessa - presupponendo l'influsso di Brunelleschi e della prima prospettiva rinascimentale. Tale lavoro di analisi e interpretazione, come suggerito più sopra, poteva essere supportato $\mathrm{e}$ allo stesso tempo condizionato dalle qualità della documentazione visiva per la ricerca - in questo caso, ad esempio, della stampa all'albumina che sappiamo essere contestuale al soggiorno fiorentino.

Non è questo il luogo di una trattazione delle fonti del pensiero warburghiano sull'espressione. Tuttavia, seguendo la traccia di materiali 'fondativi', Hilfsmittel e 'metadati', è opportuno menzionare almeno due volumi e alcune immagini. A partire da questi mesi Warburg raccoglie sistematicamente note, diagrammi, schizzi per una "teoria dell'espressione" o, secondo le varianti, "psicologia monistica dell'arte" o "filosofia psicologica dell'arte" ${ }^{31}$. Tra i testi di riferimento di questo filone di ricerca, come è noto, vi sono Mimik und Physiognomik (1867) di Theodor Piderit e The Expressions of Emotions in Man and Animals di Charles Darwin (1872) ${ }^{32}$.

Warburg timbra e segna con l'annotazione "Firenze dicembre 1888" la propria copia del volume di Piderit, nell'edizione 1886 (fig. 5). Il libro è illustrato da 94 tavole fotolitografiche, tra le quali campeggia la stampa del disegno al tratto dal Laocoonte vaticano, nel dettaglio dell'espressione del volto (fig. 6). 
Già nel novembre del 1888, alla Biblioteca Nazionale di Firenze, Aby Warburg aveva letto il libro di Darwin, traendone diversi appunti: una nota sulla utilità di questa lettura dai diari del 1888 è trascritta sul frontespizio del libro nell'ottobre 1924, in un momento importante nella biografia dello studioso, al rientro da Kreuzlingen (fig. 7) ${ }^{-33}$. Dopo Firenze, Warburg si sarebbe procurato una copia dell'edizione tedesca, segnando il volume, secondo il procedimento consueto, “Amburgo 1889". L'opera di Darwin rappresenta un esempio precoce di libro scientifico illustrato fotograficamente ${ }^{-34}$. Il procedimento fotomeccanico in questione - heliotype (collotipo o Albertype) - variante della fotolitografia, era stato inventato negli anni Cinquanta, industrializzato a fine anni Sessanta e perfezionato proprio in quegli anni da Ernst Edwards, un fotografo londinese noto a Darwin ${ }^{-35}$; permetteva di stampare fotografie in serie su carta non fotografica, rilegabile con le pagine di testo, ottenendo una riduzione notevole dei tempi e dei costi rispetto all'inserimento manuale di fotografie incollate sulle pagine tipografiche. Ciò permise a Darwin, per esempio, di corredare la propria opera, oltre che con disegni e incisioni, con fotografie tratte dalla propria collezione di studio. Tra queste, le celebri riprese di Oscar Gustave Rejlander, supporto cruciale della ricerca di Darwin; o "some excellent negatives of crying infants" - ${ }^{36}$ ottenute in prestito dal fotografo di Amburgo Kindermann, assai celebre per i ritratti cartes de visite ${ }^{-37}$. Nelle pagine di Darwin dedicate all'espressione del dolore, Laocoonte è presente come esempio di errore anatomico nella resa artistica dei muscoli facciali ${ }^{\mathbf{3 8}}$. Nell'introduzione al testo, Darwin lamenta l'inattendibilità delle opere d'arte votate al bello come fonti per lo studio sulle espressioni, spesso antiestetiche - con riferimento, in questo passaggio, proprio al Laokoon di Lessing - e veicolate piuttosto grazie all'abilità nella resa espressiva di elementi accessori della composizione ${ }^{-39}$.

Il primo capitolo dell'edizione del 1886 del volume di Piderit in possesso di Warburg include, a sua volta, una bibliografia aggiornata che acquisisce la trattazione di Darwin. In questa, e in altre sezioni del volume, leggiamo considerazioni puntuali sul valore documentale delle opere d'arte, così come su qualità e limiti dei procedimenti di stampa fotomeccanica e delle fotografie ai fini dello studio delle espressioni, ad esempio per quanto concerne i ritratti:

Wesentlich zuverlässiger als die gemalten Portraits sind natürlich die Photographien, aber selbst diese sind keineswegs immer so ähnlich wie man glauben sollte. Jeder wird die Erfahrung gemacht haben, dass verschiedene Photographien desselben Menschen sich oft wenig gleichen, und dass man einen Bekannten manchmal erst nach längerem Besinnen in seiner Photographie wiedererkennt. [...] Dazu kommt, dass kein photographisches Portrait, wenn es en face genommen wird, ganz ähnlich werden kann, denn die Perspektive wird auf dem Bilde eine andere als sie uns in der Natur erscheint $-{ }^{40}$. 


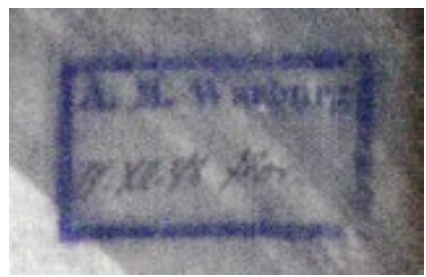

a.

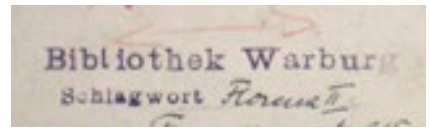

d.

Dr: A.Warburg

114 Heilwigstrasse

Tel. Gr. V, 3340.

b.

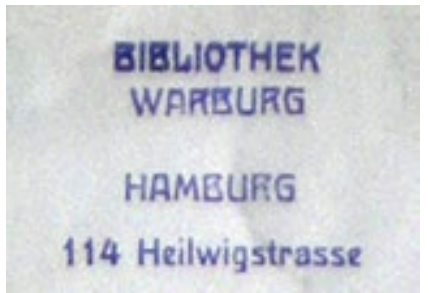

e.

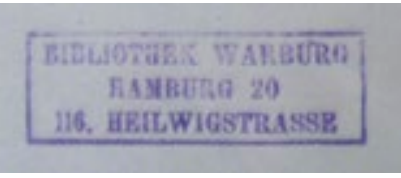

h.

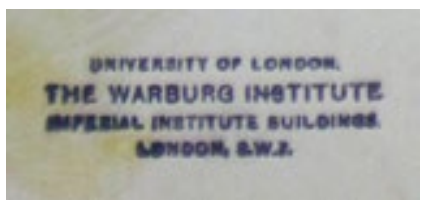

k. g.

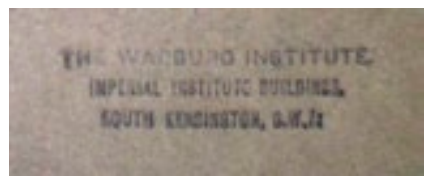

j.
OOHEX WAPPITC

HAMBURG 20 Dheo HIILWIGSTuse.

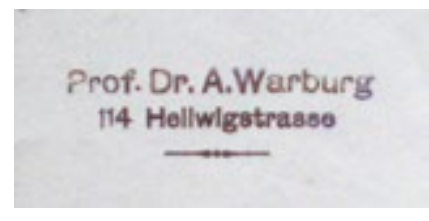

c.

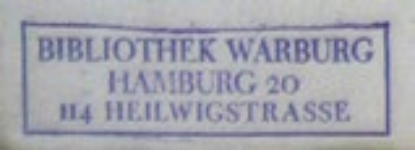

f.

08

Cronologia dei principali timbri usati per le fotografie della

Photographic Collection del Warburg Institute di Londra.

a. Post (1886) 1888-1889

[(Jahrestabellen di acquisizioni) seminario di Schmarsow a Firenze].

b. “Dr. A. Warburg / 114 Heilwigstrasse / Tel. Gr.
V, 3340." Timbro a inchiostro, post 1893 [Habilitation].

c. Post 1912 [Nomina di professore all'Università di Amburgo].

d. Post 1921

[formalizzazione della Bibliothek Warburg].

e., f. Post 1921

[formalizzazione della Bibliothek Warburg], pre 1926

[completamento dell'edificio al $116 \mathrm{di}$ Heilwigstrasse].

g. Post 1926

[completamento dell'edificio al $116 \mathrm{di}$ Heilwigstrasse].

h. Post 1926 [completamento del trasferimento della biblioteca nell'edificio al 116 di Heilwigstrasse].

i. Post 1933 [trasferimento a Londra della biblioteca].
TME WARBURG INBTTUTE

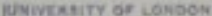
THE WARBUDO INSTITUT: cesuren sovake, Lesibon wCin ses

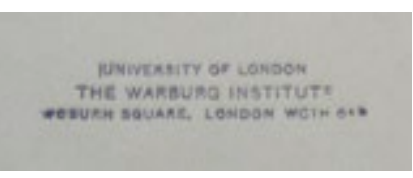

I. 
Sappiamo che Warburg, secondo una visione non estetizzante dell'arte, troverà non solo negli elementi accessori, ma proprio nell'analisi di gesti, espressioni e fisionomie uno strumento per interrogare il rapporto degli artisti con l'antico ‘dionisiaco' e il realismo moderno. Infine, documenti visivi cruciali di questo discorso warburghiano, assieme ai ritratti più sopra menzionati, saranno proprio postura e volto del Laocoonte vaticano. Così vediamo ad esempio nelle diapositive per la conferenza Der Eintritt des antikisierenden Idealstyls in die Malerei der Frührenaissance che Warburg tenne nel 1914 al Kunsthistorisches Institut a Firenze ${ }^{-41}$, o nel pannello di fotografie, numero 19, della cosiddetta "erste Fassung" -42 - databile maggio 1928 - del Bilderatlas di Mnemosyne, la grande opera lasciata incompiuta da Warburg.

Basti questo, per ora ${ }^{-43}$. Pur senza addentrarsi nelle problematiche della metodologia warburghiana, in questi esempi vediamo - letteralmente - libri e fotografie, insieme, come strumenti della ricerca e fondamenta della biblioteca che verrà. Si tratta di una precoce istruzione sulla prassi della ricerca, un precipitato materiale del binomio euristico warburghiano Wort-Bild, parola-immagine.

Ho potuto studiare

le collezioni della

Photographic Collection del

Warburg Institute nel corso

di diverse missioni di ricerca

e particolarmente come

"Grete Sondheimer" Fellow

al Warburg Institute (2010)

e British Academy Fellow

alla School of Advanced

Study, University of London

(2011-2012); ringrazio lo

staff della Photographic

Collection e dell'archivio

del Warburg Institute per

i consigli e il supporto alla

ricerca. Diversi passaggi di

questo articolo sono stati

discussi con Tiziana Serena,

che ringrazio per i puntuali

commenti.

Come corollario si pubblica in fig. 8 una cronologia dei principali timbri usati per le fotografie della

Photographic Collection del

Warburg Institute di Londra,

a partire dall'esempio

discusso più sopra e fino

alla data di completamento

dell'attuale edificio londinese di Woburn

Square.

$-{ }^{1}$ Tra i primi titoli su questo dibattito, si vedano il saggio Freitag 1979, i contributi raccolti in Roberts 1995 e il lavoro seminale Hamber 1996. Un ampio aggiornamento di prospettiva sul tema e relativa bibliografia critica è in Caraffa 2011; si veda anche Caraffa / Serena 2012.

$-{ }^{2}$ Cfr. Cestelli

Guidi / Mann 1998 e

Bredekamp 2019.

-3 Sulla fotografia come strumento e medium della storia dell'arte vedi Caraffa 2009.

-4 Le notizie

relative alla storia del

Kunsthistorisches Institut di Firenze sono tratte da Hubert 1997.

- 5 "Kunstchronik" 1894, p. 201: “I'acquisizione di una biblioteca storico-artistica il più possibile completa $\mathrm{e}$ di una grande raccolta di illustrazioni adatte a studi comparativi, entrambe collocate in idonei locali e rese accessibili a una comoda utilizzazione". Qui e altrove, ove non diversamente indicato, la traduzione è mia.

$-{ }^{6}$ Un primo riferimento essenziale sulla storia della Biblioteca Warburg è la memoria di Fritz Saxl del 1944 pubblicata in Gombrich 1970.

- 7 II nome dell'istituzione

è in relazione alla costruzione dell'edificio per la biblioteca accanto a quello di residenza di Warburg. Su questo tema: Settis 1985/2010 e tra i titoli recenti, il numero monografico $\mathrm{La}$ Kulturwissenschaftliche Bibliothek Warburg comme laboratoire, in “Revue Germanique Internationale" 2018. Sugli apparati della KBW e la fotografia vedi: Wolf 1999; e il numero monografico The Warburg Haus: 
Apparatus, inscription, data, speculation, in "Philosophy of Photography" 2017. - 8 \#Warburg 1888-1918 e cfr. \#Warburg 19191926, con Jahrestabellen fino al 1925-1926, ossia fino al trasferimento nel nuovo edificio al 116 di Heilwigstrasse, nel distretto Harvestehude ad Amburgo. Alcuni di quest diagrammi sono pubblicati in Stockhausen 1992.

Il catalogo dell'archivio del Warburg Institute è in corso di revisione; le segnature qui indicate sono pertanto da ritenere provvisorie.

- 9 \#Warburg 07.01.1889:

“Debbo gettare le fondamenta della mia biblioteca e collezione di fotografie; entrambe costano molto e rappresentano un bene duraturo".

- 10 \#Warburg 27.01.1889:

"debbo avere a portata di mano tutti gli strumenti (libri, fotografie)".

- ${ }^{11}$ Per una bibliografia sulla storia della KBW e il suo trasferimento a Londra si vedano $i$ contributi raccolti in "Common Knowledge" 2012 e Fleckner / Mack 2015. - 12 Per una storia della collezione fotografica del Warburg Institute rimando a Mazzucco 2012. -13 Le tre fotografie citate risultano acquisite a Firenze nell'inverno 1888-1889. Su questo e altri timbri della collezione cfr. la fig. 8 e didascalia relativa.

$-{ }^{14}$ Un riferimento essenziale per la storia 'tecnologica' della fotografia è Gernsheim / Gernsheim 1969.

- ${ }^{15}$ Si veda Wölfflin 1897 e Wölfflin 1915 e cfr. Cestelli Guidi 2008 e Johnson 2013. Sulla fotografia della scultura vedi tra i suoi precoci contributi Bergstein 1992 oltre a Johnson 1998.

$-{ }^{16}$ Su Warburg e la fotografia come "aidemémoire" e "inconscio ottico" cfr. Pollock 2011. $-{ }^{17}$ Per un primo orientamento sul tema si veda Fawcett 1986.

- ${ }^{18}$ Cfr. Naya 1870.

$-{ }^{19}$ Cfr. Filippin 2009 e Filippin 2011.

$-{ }^{20}$ Warburg 1902. Sul

"montaggio" di questo saggio warburghiano e le relative illustrazioni si veda Michaud 1998, pp. 97-119. $-{ }^{21}$ Warburg 1902, p. 13: "ripresa di dettaglio di formato grande".

- 22 Warburg 1902,

p. 12, n. 2: “Die auf

Taf. II-V publizierte

Detailaufnahmen haben

Gebr. Alinari auf meine

Veranlassung zum ersten Male angefertig.

Abbildungen 1-5 nach bereits vorhandenem

Photographien der Gebr.

Alinari".

- 23 \#Warburg 03.12.1901-

21.01.1902. A febbraio

Warburg invia a Heinrich

Brockhaus, direttore del

Kunsthistorisches Institut in Florenz, venti copie del saggio Bildniskunst con indicazione dei relativi destinatari: agli Alinari vanno due copie (\#Warburg 13.02.1902). -24 Un saggio classico sul tema dell'impatto della fotografia su comunicazione $e$ percezione visiva è Jussim 1974.

- 25 Alinari 1876, p.

107: “6908 [piccola: $18 \times 25 \mathrm{~cm}$ ] II Martirio di San Filippo, lunetta sopra il suddetto [6907 San Giovanni resuscita Drusiana]"; debbo a Francesca Mambelli questa identificazione. Sui cataloghi delle ditte fotografiche, si veda
Cavanna / Mambelli 2019; e cfr. la mostra on-line (dal 25 novembre 2019): Trésors d'art à la portée de tous" - I cataloghi dei fotografi, a cura di Andrea Bacchi, Costanza Caraffa, Almut Goldhahn e Francesca Mambelli, in <http://photothek. khi.fi.it/documents/ oau/00000309> (10.04.2020).

$-{ }^{26}$ Ferretti 1977 e Ferretti 2002.

$-{ }^{27}$ Nella corrispondenza warburghiana si trova menzione della ditta Alinari a partire dal 1890 (\#von Térey 14.03.1890, verifica di un ordine ad Alinari non andato a buon fine), con un significativo picco di ordini di fotografie in corrispondenza (19281929) del lavoro per il Bilderatlas di Mnemosyne. Una precisazione: dopo l'esperienza del seminario del 1888-1889, Warburg tornerà a Firenze e vi prenderà residenza tra il 1897 e il 1902.

$-{ }^{28}$ Cfr. anche il

"Contobuch" dello studioso, "Ausgaben für Bücher und Photographien" (\#Warburg 1887-1904), fol. 4: “Winter 88/89 Alinari Phot. Ca. $400 "$.

- 29 \#Warburg 1888, 12.12.1888, p. 177. Ringrazio Steffen Haug per avere portato la mia attenzione su questa nota.

- 30 \#Warburg 1889.

- 31 \#Warburg 1888-95,

1901; cfr. saggi e apparati in: Warburg $2011 \mathrm{e}$ Warburg 2015.

- 32 Piderit 1886 [1867], Darwin 1872 [1872].

- 33 \#Warburg 1888. La notizia è già in Gombrich 1970. Questa nota e le due letture del testo darwiniano nel 1888 e nel 1924 sono state discusse in diverse occasioni; cfr. da ultimo l'intervento 
di Sigrid Weigel, e relativa documentazione, presentato al convegno Aby Warburg 150. Work. Legacy. Promise, London UCL Institute of Education, 13-15 giugno 2016 (in corso di pubblicazione).

- ${ }^{34}$ Una schedatura di questo tipo di materiali è accessibile dal Catalogue of Photographically Illustrated Book della British Library in <http:// www.bl.uk/catalogues/ photographyinbooks/ welcome.htm> (15.04.2020). Sulle illustrazioni nell'opera di Darwin, fotografiche e non, e il contesto della cultura visiva coeva, v. Smith 2006 e Voss 2007.

- 35 Cfr. Harrison 1872; Edwards 1876; Prodger 2009.
- 36 “Alcune eccellenti negative di bambini che piangono ["einiger ausgezeichneter Negative von weinenden Kindern]" nell'edizione tedesca della collezione Warburg: Darwin [1872] 1872, p. 25. -37 Se ne trova menzione persino nella corrispondenza di Warburg (\#Warburg 29.07.1900). - 38 Cfr. Darwin [1872] 1872, p. 186. -39 Ivi, p. 14. - 40 Piderit [1867] 1886, pp. 144-145:

“Naturalmente, le fotografie sono molto più affidabili dei ritratti dipinti, ma anche queste non sono affatto sempre verosimili come si potrebbe pensare. Tutti avranno avuto esperienza del fatto che spesso fotografie differenti della stessa persona sono molto dissimili tra loro e che a volte si riconosce un conoscente in fotografia solo dopo lunga riflessione. [...] A ciò si aggiunge il fatto che nessun ritratto fotografico, se ripreso di fronte, può essere molto somigliante, perché la prospettiva nell'immagine è differente da come ci appare in natura".

- 41 \#Warburg 1914.

- 42 \#Warburg 1928

- 43 Una più ampia trattazione di questi temi è inclusa in un mio volume in corso di pubblicazione (Terytoria Książki, Gdańsk, 2021).

Alinari 1876 Prima appendice al Catalogo Generale delle riproduzioni fotografiche pubblicate per cura dei Fratelli Alinari, Firenze, Alinari, 1876.

Bergstein 1992 Mary Bergstein, Lonely Aphrodites: On the Documentary Photography of Sculpture, in "Art Bulletin", vol. 74, n. 3, September 1992, pp. 475-498.

Bredekamp 2019 Horst Bredekamp, Aby Warburg, der Indianer. Berliner Erkundungen einer liberalen Ethnologie, Berlin, Wagenbach, 2019.

Caraffa 2009 Costanza Caraffa (a cura di), Fotografie als Instrument und Medium der Kunstgeschichte, Berlin, Dt. Kunstverlag, 2009.

Caraffa 2011 Costanza Caraffa (a cura di), Photo Archives and the Photographic Memory of Art History, Berlin-Munich, Deutscher Kunstverlag, 2011.

Caraffa / Serena 2012 Costanza Caraffa / Tiziana Serena (a cura di), Archivi fotografici. Spazi del sapere, luoghi della ricerca, numero monografico di "Ricerche di storia dell'arte", n. 106, 2012.

Cavanna / Mambelli 2019 Pierangelo Cavanna / Francesca Mambelli (a cura di), Un patrimonio da ordinare: $i$ cataloghi a stampa dei fotografi, atti della conferenza (Bologna, 2019), Bologna, Fondazione Federico Zeri / Università di Bologna, 2019.

Cestelli Guidi / Mann 1998 Benedetta Cestelli Guidi / Nicholas Mann (a cura di), Photographs at the Frontier. Aby Warburg in America 1895-1896, London, The Warburg Institute-Merrel Holberton, 1998.

Cestelli Guidi 2008 Benedetta Cestelli Guidi, Il fotografo al museo, in Heinrich Wölfflin, Fotografare la scultura, a cura di Ead., Mantova, Tre Lune, 2008, pp. 40-67. 
"Common Knowledge" 2012 The Warburg Institute. A Special Issue on the Library and Its Readers, in "Common Knowledge", vol. 18, n. 1, Winter 2012.

Darwin 1872 [1872] Charles Darwin, Der Ausdruck der Gemüthsbewegungen bei dem Menschen und den Thieren, Stuttgart, E. Schweizerbart'sche Verlagshandlung, 1872 [ed. orig. London 1872].

Edwards 1876 Ernest Edwards, The Helyotype Process, Boston, James R. Osgoood \& Co., 1876.

Fawcett 1986 Trevor Fawcett, Graphic Versus Photographic in the Nineteenth-Century Reproduction, in "Art History" vol. 9, n. 2, June 1986, pp. 185-212.

Ferretti 1977 Massimo Ferretti, Fra traduzione e riduzione. La fotografia dell'arte come oggetto e come modello, in Wladimiro Settimelli / Massimo Zevi (a cura di), Gli Alinari fotografi a Firenze 1852-1920, Firenze, Alinari, 1977, pp. 116-142.

Ferretti 2002 Massimo Ferretti, Immagini di cose presenti, immagini di cose assenti: aspetti storici della riproduzione d'arte, in Arturo Carlo Quintavalle / Monica Maffioli (a cura di), Fratelli Alinari. Fotografi in Firenze. 150 anni che illustrarono il mondo 1852/2002, Firenze, Alinari, 2002, pp. 217-238.

Filippin 2009 Sara Filippin, Carlo Naya e gli affreschi di Giotto a Padova. La prima campagna fotografica tra mercato e conservazione, in "AFT", n. 50, dicembre 2009, pp. 18-30.

Filippin 2011 Sara Filippin, “La fotografia come medium nella lettura ed interpretazione dell'opera d'arte. Carlo Naya e la Cappella degli Scrovegni di Padova", in Claudia Caramanna / Novella Macola / Laura Nazzi (a cura di), Citazioni, modelli e tipologie nella produzione dell'opera d'arte, atti delle giornate di studio (Padova, 2008), Padova, Cleup, 2011, pp. 225-235.

Fleckner / Mack 2015 Uwe Fleckner / Peter Mack (a cura di), The Afterlife of the Kulturwissenschaftliche Bibliothek Warburg: The Emigration and the Early Years of the Warburg Institute in London, in "Vorträge aus dem Warburg-Haus" n. 12, Berlin, De Gruyter, 2015.

Freitag 1979 Wolfgang Freitag, Early Use of Photography in the History of Art, in "Art Journal", vol. 39, n. 2, 1979, pp. 117-123.

Gernsheim / Gernsheim 1969 Alison Gernsheim / Helmut Gernsheim, The History of Photography from the Camera Obscura to the Beginning of the Modern Era, New York, McGraw-Hill, 1969.

Gombrich 1970 Ernst H. Gombrich, Aby Warburg. An Intellectual Biography, London, The Warburg Institute, 1970.

Hamber 1996 Anthony Hamber, Photographing the Fine Arts, Amsterdam, Gordon and Breach, 1996.

Harrison 1872 William H. Harrison, The Heliotype process, in "Nature", 1 June 1872, pp. 85-87.

Hubert 1997 Hans W. Hubert, L'Istituto germanico di storia dell'arte di Firenze, Firenze, Ventilabro, 1997.

Johnson 1998 Geraldine Johnson, Sculpture and Photography, Cambridge, UK, Cambridge University Press, 1998.

Johnson 2013 Geraldine Johnson, "(Un)richtige Aufnahme”: Renaissance Sculpture and the Visual Historiography of Art History, in "Art History", vol. 36, n. 1, February 2013, pp. 12-51. 
Jussim 1974 Estelle Jussim, Visual Communication and the Graphic Arts. Photographic Technologies in the Nineteenth Century, New York, Bowker, 1974.

“Kunstchronik" 1894 Aufruf zur Gründung eines kunstgeschichtliches Institutes, in “Kunstchronik" V. Jahrgang, 1893/94, n. 13, 25 gennaio 1894, pp. 202-203.

Mazzucco 2012 Katia Mazzucco, L'iconoteca Warburg di Amburgo. Documenti per una storia della Photographic Collection del Warburg Institute, in "Quaderni Storici", n. 3, dicembre 2012, pp. 857-887.

Michaud 1998 Philippe Alain Michaud, Aby Warburg et l'image en mouvement, Paris, Macula, 1998.

Naya 1870 Catalogo N. 1, Fotografie di Carlo Naya in Venezia, Venezia, Tipografia del Commercio di Marco Vicentini, 1870.

Piderit 1886 [1867] Theodor Piderit, Mimik und Physiognomik, Detmold, Verlag der Meyer'schen Hofbuchhhandlung, 1886 [ed. orig. Detmold 1867].

Pollock 2011 Griselda Pollock, Aby Warburg and Mnemosyne: Photography as aidemémoire, Optical Unconscious and Philosophy, in Caraffa 2011, pp. 73-97.

Prodger 2009 Philip Prodger, Darwin's Camera. Art and Photography in the Theory of Evolution, Oxford-New York, Oxford University Press, 2009.

"Revue Germanique Internationale" 2018 La Kulturwissenschaftliche Bibliothek Warburg comme laboratoire, in "Revue Germanique Internationale", n. 28, 2018, in <http://journals.openedition.org/rgi/1882> (10.05.2020).

Roberts 1995 Helen Roberts (a cura di), Art History through the Camera's Lens, Amsterdam, Gordon and Breach, 1995.

Settis 1985/2010 Salvatore Settis, Warburg continuatus. Descrizione di una biblioteca, in "Quaderni storici", vol. 20, n. 58, 1985, pp. 5-38; edizione aggiornata in Warburg continuatus. Descripcion de una biblioteca, Madrid, Ediciones de la Central, 2010.

Smith 2006 Jonathan Smith, Charles Darwin and Victorian Visual Culture, Cambridge, Cambridge University Press, 2006.

Stockhausen 1992 Tilmann von Stockhausen, Die Kulturwissenschaftliche Bibliothek Warburg Architektur, Einrichtung und Organisation, Hamburg, Dölling und Galitz, 1992.

Voss 2007 Julia Voss, Darwins Bilder. Ansichten der Evolutionstheorie, 1837-1874, Frankfurt, Fischer Taschenbuch Verlag, 2007.

Warburg 1902 Aby M. Warburg, Bildniskunst und florentinisches Bürgertum, I. Domenico Ghirlandaio in Santa Trinita. Die Bildnisse des Lorenzo de Medici und seiner Angehörigen, Leipzig, Seemann, 1902.

Warburg 2011 Aby M. Warburg, Frammenti sull'espressione. Grundlegende Bruchstücke zu einer pragmatischen Ausdruckskunde, ed. critica a cura di Sabine Müller, trad. it. di M. Ghelardi / G. Targia, Pisa, Edizioni della Normale, 2011.

Warburg 2015 Aby M. Warburg, Fragmente zur Ausdruckskunde, Warburg Studienausgabe, Band IV, a cura di Ulrich Pfisterer / Hans Christian Hönes, Berlin, De Gruyter, 2015.

Wolf 1999 Gerhard Wolf, Aby Warburg. La fotografia y su laboratorio de historia teóricocultural de la imagen, in Centro Argentino de Investigadores de Arte (a cura di), Epílogos y prólogos para un fin de siglo, atti delle “VIII Jornadas de Teoría e Historia de las Artes" (Buenos Aires, 1999), Buenos Aires, CAIA, 1999, pp. 309-322. 
Wölfflin 1897 Heinrich Wölfflin, Wie man Skulpturen aufnehmen soll, in "Zeitschrift für bildende Kunst", n. s. 7, 1896, pp. 224-228, e n. s. 8, 1897, pp. 294-297.

Wölfflin 1915 Heinrich Wölfflin, Wie man Skulpturen aufnehem soll? Probleme der italienischen Renaissance, in "Zeitschrift für bildende Kunst", n. s. 26, 1915, pp. 237-244.

Fonti archivistiche \#von Térey 14.03.1890 Gabriel von Térey a Aby M. Warburg, cartolina ms., Firenze 14 marzo 1890. London, Warburg Institute Archive (WIA), General Correspondence (GC).

\#Warburg 1887-1904 Aby Warburg, Contobuch, 1887-1904, ms. London, Warburg Institute Archive (WIA) III.19.3.

\#Warburg 1888 Aby M. Warburg, Blocknotes, diaries, 1888, ms. London, Warburg Institute Archive (WIA) III.9.4.

\#Warburg 1888-1918 Bibliothek Warburg Jahrestabellen, Aby M. Warburg, graph of overall library growth, 1888-1918, on two fols; Mary Warburg, graph showing expenses (Bucher, Bucheinband, Spesen, Illustrationen) 1888-1918, I fol. London, Warburg Institute Archive (WIA) I.4.5.7.

\#Warburg 1888-95, 1901 Aby M. Warburg, Grundlegende Bruchstücke zu einer pragmatischen Ausdruckskunde (Monistischen Kunstpsychologie), ms. e dattil., raccolta di frammenti. London, Warburg Institute Archive (WIA) III.43.1.

\#Warburg 1889 Aby M. Warburg, Entwurf zu einer Kritik des Laokoon, ms. e dattil., testo frammentario. London, Warburg Institute Archive (WIA) III.33.2.

\#Warburg 07.01.1889 Aby M. Warburg, lettera ms. e dattil. (copia) a Charlotte Warburg, 7 gennaio 1889. London, Warburg Institute Archive (WIA), Family Correspondence (FC).

\#Warburg 27.01.1889 Aby M. Warburg, lettera ms. e dattil. (copia) a Charlotte Warburg, 7 gennaio 1889. London, Warburg Institute Archive (WIA), Family Correspondence (FC).

\#Warburg 29.07.1900 Aby M. Warburg, lettera ms. a Mary Warburg, 29 luglio 1900. London, Warburg Institute Archive (WIA), Family Correspondence (FC).

\#Warburg 03.12.1901-21.01.1902 Aby M. Warburg, lettere dattil. (copie) a Viktor Schweizer, 3 dicembre 1901, 23 dicembre 1901, 3 gennaio 1902, 21 gennaio 1902. London, Warburg Institute Archive (WIA), General Correspondence (GC).

\#Warburg 13.02.1902 Aby M. Warburg, lettera ms. e dattil. (copia) a Heinrich Brockhaus, Amburgo 13 febbraio 1902. London, Warburg Institute Archive (WIA), General Correspondence (GC).

\#Warburg 1914 Aby M. Warburg, Der Eintritt des antikisierenden Idealstyls in die Malerei der Frührenaissance, dattil. e appunti ms., testo e lista immagini, conferenza al Kunsthistorisches Institut in Florenz, 20.04.1914. London, Warburg Institute Archive (WIA), III.88.1-2.

\# Warburg 1919-26 Bibliothek Warburg Jahrestabellen, varie mani, diagrammi di costi annuali, 1919-26. London, Warburg Institute Archive (WIA) I.4.5.8-13.

\# Warburg 1928 Aby M. Warburg, A[tlas]/V/28, erste Fassung, fotografie di 43 pannelli e passpartout con annotazioni ms. di Warburg et al., maggio 1928. London, Warburg Institute Archive (WIA), III.108.7.1, foll. 8-93. 